\title{
Strates
}

STRATES Matériaux pour la recherche en sciences sociales

$12 \mid 2006$

Nouvelles tensions impériales et recompositions en Europe centrale, orientale, et CEI

\section{Solidarnosc : creuset d'idées et formes du mouvement populaire}

Jean-Claude FAMULICKI

\section{OpenEdition}

Journals

Édition électronique

URL : http://journals.openedition.org/strates/1632

DOI : $10.4000 /$ strates. 1632

ISSN : $1777-5442$

Éditeur

Laboratoire Ladyss

Édition imprimée

Date de publication : 31 décembre 2006

ISSN : 0768-8067

Référence électronique

Jean-Claude FAMULICKI, «Solidarnosc : creuset d'idées et formes du mouvement populaire », Strates [En ligne], 12 | 2006, mis en ligne le 19 juillet 2007, consulté le 08 septembre 2020. URL : http:// journals.openedition.org/strates/1632 ; DOI : https://doi.org/10.4000/strates.1632

Ce document a été généré automatiquement le 8 septembre 2020

Tous droits réservés 


\title{
Solidarnosc : creuset d'idées et formes du mouvement populaire
}

\author{
Jean-Claude FAMULICKI
}

1 La naissance du mouvement social Solidarité en août 1980, son rôle dans l'évolution de la société polonaise et les conséquences du coup d'État militaire du 13 décembre 1981 permettent à coup sûr de mieux comprendre la transition polonaise à la démocratie de 1989-1990. Les conséquences imprévisibles des négociations de la Table ronde de 1989, les problèmes de nature très différente de ces dernières années ont un peu estompé cet événement capital de l'histoire polonaise contemporaine. Mais des historiens polonais (comme Andrzej Friszke, 2003) réévaluent la spécificité et l'importance de ce moment historique, à côté du rôle décisif de la perestroïka de Mikhail Gorbatchev qui créa des possibilités inexistantes auparavant ou du rôle catalyseur du premier pèlerinage du pape Jean-Paul II en Pologne en 1979.

Les prémisses de Solidarité

2 Comme au moment de la révolte ouvrière de la Baltique en 1970 et des grèves de 1976, la vague de grèves commencée en juillet 1980 fit suite à une hausse des prix des produits alimentaires et prit immédiatement une tournure plus largement politique dans le contexte communiste. De fait, aux chantiers navals de Gdansk, les grévistes réclamaient aussi la réintégration d'Anna Walentynowicz, licenciée pour avoir milité avec quelques autres pour la création de syndicats libres. Mais la leçon fut tirée des répressions de 1970 et 1976 et du soutien des opposants intellectuels du Comité de défense des ouvriers (KOR) créé en 1976. L'idée d'une représentation "autogérée ", unie et indépendante du pouvoir, des revendications ouvrières se traduisit par une organisation des grèves sur les lieux de travail occupés, sans recourir aux manifestations de rues, en fédérant les représentations des grévistes entre les entreprises et à l'échelon local de la ville ou de la région. L'enjeu programmatique du mouvement était dans la reprise des 21 revendications du Comité de grève interentreprises de Gdansk, en commençant par le droit à un syndicat indépendant et autogéré. Jusqu'au programme complet du Congrès de Solidarité de 1981, le principe d'une action concertée pour la liberté d'expression et d'organisation, la participation 
de toutes les composantes de la société aux décisions devait rester présent. L'appel adressé aux autres pays d'Europe de l'Est à rejoindre une lutte commune pour des syndicats indépendants du pouvoir se situait dans le même esprit; souvent présenté comme inutilement provocateur à l'époque, il s'avéra prémonitoire.

3 Les messes et autres manifestations religieuses propres à la tradition catholique et l'appui mesuré de l'unique institution indépendante, représentative et tolérée, l'Église, ajoutaient une forme symbolique d'un autre type, accentuant l'impression d'unanimité nationale de cette lutte contre le pouvoir. L'esprit d'union ou d'unité contre un pouvoir considéré comme étranger à un corps social qu'il prétendait représenter («eux et nous ») parcourait toutes les composantes souvent disparates, voire incompatibles, du mouvement qui émergeaient de toutes parts après des décennies de refoulement.

4 La forme d'organisation particulière du mouvement de Solidarité, ce qui fit sa force et sa faiblesse, et en tout cas sa spécificité si frappante à l'époque pour les observateurs, se développa tout au long de son existence légale, pour disparaître ensuite dans les conditions de l'état de guerre et de la clandestinité. Les relations entre les dirigeants du syndicat et la base exigeaient la transparence, et donc la circulation immédiate de l'information obtenue du pouvoir ou d'autres régions ; les réunions auxquelles tous ne pouvaient assister nécessitaient des compte rendus exhaustifs (d'où la vogue des magnétophones minicassettes chez les délégués enregistrant scrupuleusement les débats). La prise de parole, souvent pléthorique du fait du manque de pratique des débats, se pliait à des procédures appliquées à la lettre. Le contrôle des décisions prises collectivement était systématique. L'autogestion des décisions et des tâches s'accompagnait de formes de rituels spectaculaires dans une société soumise à un contrôle total du pouvoir: floraison des différentes feuilles d'entreprises, bulletins, journaux, colportés, vendus, distribués ou échangés d'un lieu ou d'une région à l'autre et soigneusement rassemblés dans les locaux syndicaux que le mouvement s'était partout approprié. Des journaux muraux réapparurent, ou encore des journaux sonores par circuits de radio internes aux entreprises, de cassettes enregistrées des réunions régionales ou nationales. À l'exigence d'information s'ajoutait la soif d'une culture indépendante du pouvoir: des universités ouvrières s'étaient ouvertes à tous, en de nombreux lieux, y compris dans des petites villes, avec l'aide d'universitaires, d'enseignants ou d'hôtes de passage. Des spécialistes souvent improvisés exposaient l'organisation juridique et les programmes des syndicats occidentaux, des analyses politiques et historiques sur différents sujets tabous jusqu'alors en Pologne populaire : Katyn et l'insurrection de Varsovie de 1944, la dissidence à l'Est, l'invasion de Prague en 1968, la répression des révoltes ouvrières de 1956, 1970 et 1976.

5 La présence des experts et des conseillers du syndicat aux côtés de Lech Walesa et des responsables régionaux siégeant dans les instances de décision témoignait d'une alliance des travailleurs et des intellectuels contre le pouvoir mais donna lieu, dès le début, à des malentendus et à des tensions. La méconnaissance mutuelle et les reproches de deux milieux rarement unis dans les mobilisations passées, n'étaient que progressivement surmontés. Il s'y ajoutait une compréhension souvent divergente de l'«autolimitation » du mouvement face aux risques de répression ou d'intervention soviétique ou encore des formes d'intervention et de mobilisation propres à maintenir la cohésion et la modération de ce mouvement, les conseillers privilégiant le dialogue direct avec Lech Walesa et l'influence sur la base à travers lui. Des conceptions ou des références diverses coexistaient, allant d'intellectuels connus comme proches des 
groupements ou publications catholiques tolérés ou encore de l'épiscopat à ceux de la mouvance laïque et sociale du KOR. Cette dernière allait décider de se dissoudre officiellement dans le mouvement, mais jusqu'à la Table ronde de 1989 elle devait incarner le danger principal du mouvement, pour les dirigeants communistes aussi bien que pour les "vrais" Polonais catholiques de Solidarité, allergiques à son influence.

6 L'Union soviétique se bornant à menacer d'une intervention sans intention d'y recourir, comme on le sait à présent, le pouvoir communiste choisit, dès les premiers temps de Solidarité, de préparer secrètement l'épreuve de force, pour maintenir le plus longtemps possible sa stratégie du refus de toute mise en cause de son monopole. Les formes spécifiques d'organisation et de revendication de Solidarité l'incitèrent à une série de manœuvres et de divisions, depuis la crise de Bydgoszcz de mars 1981 jusqu'aux atermoiements au moment décisif de la transition, entre les deux vagues de grèves d'une nouvelle génération d'ouvriers en 1988 et l'ouverture des négociations de la Table ronde de 1989.

De l'action publique aux restrictions de la clandestinité

7 L'effet de surprise de l'instauration en toute illégalité socialiste de l'état de siège le 13 décembre 1981 devait jouer à plein dans un mouvement éprouvé par les divergences et la lassitude de la population en butte aux restrictions économiques. Faute de décisions pour se préparer à une action concertée de protestation, (sauf dans le bastion de Basse Silésie où les mobilisations avant, pendant et après l'état de guerre furent très souvent les plus résolues), le fonctionnement ouvert et démocratique de la période légale livra locaux, structures et militants aux coups d'une répression violente et programmée. Contrairement aux justifications successives, cette répression du mouvement de Solidarité ne fut pas qu'un moindre mal. Les arrestations et internements concernèrent 10000 personnes sur toute la période, auxquelles s'ajoutent les victimes : les neuf grévistes de la mine de Wujek, à Katowice en décembre 1981, tués par balles, et les autres nombreux décès dans les premières grèves de protestation, les manifestants tués par les forces de répression dans les mobilisations ultérieures, comme en août 1982, à Lubin, près de Wroclaw, les meurtres inexpliqués de militants en province à de nombreuses reprises ou les assassinats élucidés et publiquement réprouvés, comme ceux du lycéen Gregorz Przemyk ou du père Popieluszko.

8 La suppression des libertés de mouvement et d'information ne fut compensée que dans une très faible mesure par la principale activité du syndicat délégalisé : l'édition et la diffusion d'un nombre encore plus élevé qu'auparavant de publications clandestines, plusieurs milliers de titres de périodiques différents et autant de titres d'ouvrages publiés par les officines d'édition illégales. Les décisions prises par les différentes instances du syndicat dans les conditions de la clandestinité et jusqu'à l'ouverture des négociations de 1989 furent l'objet d'incessantes mises en cause, à l'intérieur du syndicat comme par des organisations dissidentes, telle Solidarité Combattante, dès 1982. L'absence d'unité ou d'adhésion aux décisions s'ajouta aux effets de la répression et marginalisa fortement le mouvement dans une société polonaise confrontée à des conditions de vie et d'approvisionnement dégradées. Les consignes de boycott ou de mobilisation rencontraient très peu d'écho après 1983; le nombre de personnes réceptives aux idées de Solidarité passa de 9 millions d'adhérents de 1980 à quelques centaines de mille au creux des années 1986-1987 pour atteindre un ou deux millions entre 1988 et 1989, nettement moins que les adhérents de l'OPZZ, syndicat 
gouvernemental, seul habilité à représenter les travailleurs dans les entreprises; les fortes abstentions au référendum de 1987 ou, après la transition, aux élections législatives de juin 1989 en portaient aussi la trace. L'absence de vrais débats, ceux des publications radicalisant les divergences par manque d'enjeux tangibles, venait après le choc de la répression par le pouvoir communiste de la volonté de dialogue. Cela favorisa en retour, dans la presse clandestine, le développement des idées libérales, les idées sociales ou de gauche étant assimilées à celle du pouvoir ou taxées de dangereuses utopies, ce qui réduisit d'autant la réflexion sur la complexité de la situation polonaise.

L'influence de l'Église, circonscrite au départ, se développa dans la nouvelle situation. Le pouvoir comptait sur les appels à la modération et sur le sens des responsabilités de la hiérarchie catholique, tentant dès le début de l'état de guerre jusqu'à la Table ronde de dissocier l'opposition responsable, qui en était proche, des "extrémistes » du syndicat et d'isoler ses dirigeants. Après le choc de l'état de guerre, la suppression des libertés, l'internement de la plupart des dirigeants syndicats de tous les échelons, et la militarisation de l'économie, une propagande virulente rendait les militants de Solidarité responsables du chaos économique, les présentant comme des ennemis de la nation responsables des affrontements et de leurs conséquences. Du fait de l'absence de perspectives de mobilisation ou des risques encourus qui limitaient les activités clandestines, les églises devenaient les seuls lieux publics de manifestation d'indépendance à l'égard du pouvoir, par les messes pour la patrie ou les rassemblements qui en étaient l'occasion, hébergeant la distribution de l'aide internationale comme les manifestations de la culture chrétienne tolérées, où pouvait s'exprimer la référence à Solidarité. Beaucoup d'opposants, d'intellectuels, firent état de leur ferveur retrouvée. Les représentants de l'épiscopat multiplièrent les initiatives et les déclarations. On vit se renforcer les courants nationalistes catholiques qui ne s'étaient signalés que par des excès de chauvinisme ici ou là dans la première Solidarité (la "Grande", comme on l'a appelée, après avoir assisté aux déchirements d'après 1990). Nombre de dirigeants clandestins déploraient qu'il fût plus difficile de mobiliser les militants pour des activités clandestines en faveur de la relégalisation de Solidarité que pour arborer le $\mathrm{V}$ de la victoire promise à la sortie des offices. Les pèlerinages de Jean-Paul II en 1983, puis en 1987, malgré les polémiques sur la proscription des banderoles du syndicat dans les cortèges, renforcèrent cette présence de l'Église dans la vie publique, parfois au détriment de l'état d'esprit antérieur.

De 1986 à 1988, l'effervescence qu'avait connue la société polonaise semblait définitivement disparue et la confrontation du pouvoir et du syndicat, dans l'impasse. Des déclarations isolées, l'annonce de structures de direction syndicales « légales » ou de comités de réflexion auprès de Lech Walesa n'avaient pas autant d'écho que l'activité multiforme d'une nouvelle génération de contestataires: les jeunes lycéens et apprentis de la contre-culture rock, les étudiants radicalisés du NZS (Niezalezne Zrzeszenie Studentów [Association indépendante des étudiants]), les pacifistes défendant l'objection de conscience, l'Alternative Orange à Wroclaw tournant le pouvoir en dérision dans des happenings de rue de plus en plus massifs. L'ampleur du marasme économique et une chute de popularité catastrophique du pouvoir dans les sondages conjuguèrent alors leurs effets en 1988 avec deux vagues de grèves contre des augmentations de prix, animées dans tout le pays, par de jeunes ouvriers qui réclamaient la reconnaissance de Solidarité. 
11 Aux négociations de la Table ronde de 1989 qui suivirent, dénoncées par les courants syndicaux hostiles au "compromis historique», mais aussi dans les réunions préparatoires en cercles plus restreints, l'épiscopat offrit ses bons offices, entre le pouvoir affaibli et Solidarité renaissante qui posait comme préalable sa relégalisation. Les dirigeants communistes tentèrent une ultime fois d'obtenir la mise à l'écart de participants indésirables à leurs yeux, comme Jacek Kuron ou Adam Michnik et de diluer l'identité syndicale dans une « union nationale » qu'ils estimaient indispensable face aux échéances politiques et aux pénibles décisions économiques à venir. Les généraux de l'état de siège eurent in fine à convaincre les conservateurs de leur parti, hostiles à un compromis avec une opposition si souvent disqualifiée. De son côté, aux élections de juin 1989 qui suivirent, dans quelques cas, l'épiscopat adouba sans succès des candidats qui se présentaient contre ceux du syndicat, responsables ou conseillers. Pour certains observateurs avertis de la scène politique polonaise, tel Jerzy Giedroyc, le fondateur et directeur de la revue Kultura, le rôle de l'Église dans la sortie de la Pologne du communisme a pu se trouver rétrospectivement surévalué à la lumière des appréciations favorables de son action dans la société polonaise après le 13 décembre 1981.

\section{BIBLIOGRAPHIE}

Beauvois D. et Famulicki J. C., (dir.), « La Pologne d'Est en Ouest 1945-2001 », Matériaux pour l'histoire de notre temps, $\mathrm{n}^{\circ}$ 61-62, 2001.

Friszke A., Polska. Losy panstwa i narodu 1939-1989, Warszawa, Wydawnictwo Iskry 2003, 519 p.

Le programme de Solidarnosc au congrès de Gdansk en 1981, 1982, PUL, 58 p.

\section{RÉSUMÉS}

L'auteur restitue le mouvement Solidarnosc dans son contexte historique, donne un aperçu de son mode de fonctionnement et tente de lui redonner la place novatrice et originale qui lui revient comme mouvement social spécifique, autolimité, dans un contexte communiste répressif qui a su dans sa première phase faire aboutir ses revendications démocratiques en faveur d'un syndicat et d'une presse libres et indépendants tout en restant dans le cadre ouvrier permis et valorisé par le régime et atteindre ainsi un niveau de mobilisation inattendu dans le contexte des années 1980. Il montre aussi ses limites et ses faiblesses liées aux divisions internes qui ont fait à la fois sa richesse et sa dynamique mais aussi sa perte, et facilité sa récupération par une Église polonaise offensive, soutenue de l'extérieur par une autorité pontificale décidée à en finir avec le système communiste. Ce mouvement a néanmoins été et reste un modèle pour les nouvelles démocraties du monde post-soviétique qui s'en nourrissent et s'en inspirent pour en finir avec les séquelles d'un système qu'elles tentent d'éradiquer aujourd'hui de leur vie politique.

Jean Claude Famuliki. Solidarnosc: a crucible for ideas and forms of popular movement 
The author provides an insight in the way the Solidarity movement functioned and attempts to give it back the innovative and original place it deserves as a self-limited specific social movement in a repressive communist context. It was able in its first phase to succeed in its democratic claims for a free, independent trade-union and a free press whilst staying within the boundaries of the workers' framework, one which was allowed and valued by the regime, thus getting an unexpected amount of mobilisation in the context of the 1980's. He also shows the movement's limitation and weaknesses, linked to the internal divisions which made its richness and dynamism but also caused its downfall, making it easy for a Polish church on the offensive to take it over, supported as it was from the outside by a papal authority who wanted to finish with communism once and for all.

\section{AUTEUR}

\section{JEAN-CLAUDE FAMULICKI}

Conservateur, responsable du Musée d'histoire contemporaine et du fonds polonais de la BDIC, jean-claude.famulicki@bdic.fr 